\title{
Simple schizophrenia or disorganisation syndrome? A case report and review of the literature
}

\author{
Susannah Whitwell, Jessica Bramham \& John Moriarty
}

Abstract Simple schizophrenia is a sometimes controversial diagnosis. Here we review the concept with particular reference to its history in diagnostic systems. Using an illustrative real case of a 25-year-old man, we show that there is a need to retain this diagnostic category, which may fit better within proposed dimensional (psychomotor poverty, disorganisation and reality distortion) rather than categorical classifications of schizophrenia. Symptoms of the disorder may be better revealed by functional assessment than by relying on descriptive psychopathology.

Vignette: Mr B

Mr B is a 25-year-old man, referred to a national neuropsychiatry assessment service by his community mental health team. The referral was at the request of his parents, who wondered if he might have Asperger syndrome.

Mr B was born by normal vaginal delivery at term and his parents described normal motor, language and social communication milestones. He grew up with both parents and three brothers. Throughout primary and secondary mainstream school he made friends easily and excelled at sport to the extent that at age 11 he gained a black belt in karate. Mr B left school after achieving an average performance in state examinations, on a par with his brothers' achievements. School reports are positive and describe no behavioural or academic problems.

However, at age $15 \mathrm{Mr} \mathrm{B}$ began to be more isolated, losing contact with school friends and interacting minimally with his family, eating in his bedroom alone and maintaining poor personal hygiene.

Over the next 10 years Mr B had little contact outside the family home. He attempted several jobs, including labouring and factory work, but despite an initial good impression was asked to leave each position, often after a very short period. Reasons included an inability to conform to safety regulations, lateness and chaotic behaviour. At the time of admission he had not worked at all during the previous year.
Mr B revealed increasing difficulties in planning and making decisions. An example given by his parents was an occasion when he attempted to cut a piece of wood with an electric saw while balancing the wood over banisters. His parents became so concerned both by the dangerousness of this and his aggressive response when they asked him to stop that they called the police. Mr B was reported as being increasingly irritable. If his parents thwarted his actions, episodes of verbal aggression were precipitated.

The deterioration in overall functioning, social behaviour and planning ability was gradual and $\mathrm{Mr}$ B's parents sought help after 10 years only because of his low threshold for verbal aggression and his inability to hold down a job. For many years his social withdrawal was attributed by the family to selfconsciousness related to his facial acne.

There was no family history of psychiatric disorder, no medical history and up to admission Mr B had lived with his parents.

Psychiatric history Mr B's first psychiatric assessment was by his local community mental health team (CMHT). It was thought that his symptoms might reflect dysmorphophobia, although particular somatic preoccupations were not elicited. He was prescribed paroxetine, which did not alter his mental state. Five months later, Mr B was referred to the neuropsychiatry service for a second opinion.

Susannah Whitwell trained in psychiatry at the Maudsley Hospital, London, and she is now a medical officer in psychiatry at Waitakere Hospital, Auckland, New Zealand. Jessica Bramham is a clinical psychologist with South London and Maudsley NHS Trust, London, where she has worked for 4 years in the neuropsychiatry and behavioural genetic disorders services. She is involved in researching social cognition following neurosurgery, and attention-deficit hyperactivity disorder in adults. John Moriarty is a consultant psychiatrist with South London and Maudsley NHS Trust (Department of Psychological Medicine, King's College Hospital, London SE5 9RS, UK. E-mail: john.moriarty@slam.nhs.uk). He has worked in community services in central London and now provides a neuropsychiatry service. His research interests include psychiatric aspects of epilepsy and movement disorders. Mr B gave his written consent for this article to be published. 
Mental state examination Examination revealed a young White man, slightly unkempt, able to maintain good eye contact and rapport, but who appeared perplexed. Mr B struggled to describe his experience of feeling different from other people and his sense of confusion and muddled thoughts. It was sometimes difficult to follow his train of thought and he used stock phrases such as 'I just want to get on with life'. He reported low mood attributed to his sense that his life was going badly. He did not experience suicidal thoughts, sleep and appetite were unchanged, and objectively $\mathrm{Mr} \mathrm{B}$ was euthymic. There was no evidence of hallucinations or delusions. He was not apathetic and was felt to have a warm and reactive affect.

Physical examination This was unremarkable, with no evidence of focal neurological signs, movement disorder or soft neurological signs. Investigations included routine blood tests, which were within normal ranges, an electroencephalogram, which was normal, and magentic resonance imaging (MRI) of the head, which revealed a $13 \mathrm{~mm}$ pineal cyst.

Cognitive functioning A neuropsychological assessment indicated that his current general intellectual functioning fell at the bottom of the average range (full-scale IQ = 90) as measured by the Wechsler Adult Intelligence Scale - Third Edition (WAIS-III; Wechsler, 1997). This represents a decline from premorbid levels, estimated using the Wechsler Test of Adult Reading (WTAR; Wechsler, 2001) to fall in the middle of the average range (full-scale $I Q=102$ ). This discrepancy is statistically significant although relatively frequent, occurring in $10-24 \%$ of the normal population (Wechsler, 2001).

Mr B exhibited significant memory impairments. His working memory index (WMI) score from the WAIS-III $($ WMI $=80)$ had declined from estimated premorbid levels. Assessment with the Adult Memory and Information Processing Battery (Coughlan \& Hollows, 1995) revealed verbal memory deficits in both learning and recall, although his visual memory was unimpaired.

Tests evaluating Mr B's language functioning included the Vocabulary and Similarities sub-tests of the WAIS-III, the WTAR and Thurstone's Word Fluency Test (Lezak, 1995). His vocabulary and reading abilities remained in keeping with levels predicted by his demographic background. In contrast, he showed deterioration in verbal abstract reasoning, as indicated by a poor score on the Similarities sub-test. He also had difficulties on Thurstone's Word Fluency task, where his performance fell below the 10th percentile, indicating a deficit in spontaneous word generation.

Executive functioning $\mathrm{Mr} \mathrm{B}^{\prime}$ s executive functioning was assessed with Thurstone's Word Fluency Test, the Behavioural Assessment of Dysexecutive Syndrome (Wilson et al, 1996) and the Hayling and Brixton Tests (Burgess \& Shallice, 1997). Mr B performance was impaired on most tests, indicating deficits in verbal fluency, strategy formation, planning, organisation, response inhibition and rule learning. Further evidence consistent with executive dysfunction included behavioural disinhibition during testing, including telling a dirty joke and being unaware that it was inappropriate, and being undressed when testing was to be resumed after a break.

Occupational therapy assessment An occupational therapy assessment was undertaken using the Assessment of Motor and Process Skills (Fisher, 2003). This is an observational assessment enabling simultaneous evaluation of motor and process skills and their effect on a person's ability to perform domestic or personal daily living skills required for community living. The results are analysed to provide profiles of performance and measures of motor and process skills.

Given a range of options, $\mathrm{Mr}$ B chose to be assessed on making a fresh fruit salad and beans on toast (harder than average tasks). His motor skills were satisfactory and were deemed to not limit his safety, although he had some stability problems in moving around the kitchen and he dropped some objects when using both hands. In contrast his process skills were well below the cut-off for satisfactory performance. He demonstrated consistent problems with temporal organisation and difficulties in initiating steps and frequently started a new task before completing the previous one. He left steps out, repeated steps or put them in the wrong order, such as buttering the bread before putting it in the toaster. However, despite his difficulties, he was motivated to participate in the assessment and was enthusiastic about becoming more independent.

Diagnosis, treatment and outcome Mr B's clinical picture was therefore characterised by progressive though insidious development of odd behaviour, difficulties meeting the demands of society and decline in overall performance. In the absence of hallucinations and delusions over a 10 -year period, this is consistent with a diagnosis of simple schizophrenia as defined in ICD-10 (World Health Organization, 1992). We prescribed olanzapine. This was discontinued after 1 month because of intolerable side-effects of weight gain and drowsiness. Risperidone was initiated with few side-effects, and Mr B reported an improvement in the subjective clarity of his thoughts. His parents reported satisfaction that their son appeared calmer and less impulsive during a period of leave in the family home. Mr B was discharged home and to the care of the local CMHT with a plan to attend a local resource centre daily, with regular community psychiatric nurse and psychiatrist contact.

A year later, Mr B remained engaged with his local CMHT and had just begun a semi-residential occupational and social rehabilitation programme. He continued to be disorganised, but showed less impulsivity and was generally calm. He remained on risperidone. 


\section{Discussion}

Mr B's case illustrates the presentation of simple schizophrenia characterised by a gradual insidious deterioration, leading, in this case, to a 10-year delay in diagnosis and treatment. Interestingly, the extent of Mr B's difficulties was less readily revealed by the descriptive psychopathological assessment and more obviously determined by the neuropsychology and occupational therapy functional assessments.

A diagnosis of simple schizophrenia can only be made after other causes of deterioration have been excluded, specifically the cognitive impairment of early-onset dementia, learning disability (ICD-10 mental retardation) or a pervasive developmental disorder, which could present with similar difficulties. Mr B's clearly documented good premorbid level of functioning both academically and socially excluded a pervasive developmental disorder such as Asperger syndrome or learning disability. The absence of an aetiological medical condition and the absence of findings on MRI made the diagnosis of dementia in a young person extremely unlikely.

\section{Simple schizophrenia: history of the concept}

Simple schizophrenia (dementia simplex) was first described by Otto Diem in 1903 as a subgroup of Emil Kraepelin's dementia praecox:

\footnotetext{
'In addition to the clinical pictures... which terminate in ... dementia praecox, ... [there is] a further type leading to the same terminal state,... but [for which] the onset is simple, indolent without special prodrome, without acute episodes, without illusions or hallucinations, ...' (quoted in Black \& Boffeli , 1989).
}

This syndrome was elaborated by Eugen Bleuler in 1908. He viewed simple schizophrenia as a syndrome characterised by the core features of schizophrenia: autism, loosening of associations, ambivalence and affective disturbance but lacking the accessory symptoms of hallucinations and delusions. Bleuler commented that this picture was seen more commonly outside institutions, for example in labourers, vagrants and peddlers (Bleuler, 1911, cited in Black \& Boffeli, 1989).

In 1913 the diagnostic category was further recognised when Kraepelin himself added simple schizophrenia as a subtype of schizophrenia in the eighth edition of his textbook of psychiatry (Kraepelin, 1919). Diem, Bleuler and Kraepelin all agreed that simple schizophrenia was characterised by a change in personality, a loss of interests and an overall loss of functional ability. This state progressed to a clinical picture shared with the other subtypes of schizophrenia of avolition, amotivation and inactivity. The diagnosis received widespread acceptance, as evidenced by its inclusion in standard textbooks and psychiatric literature, and in 1948 it was added to the sixth edition of what has become the International Classification of Diseases (ICD) (World Health Organization, 1948). In 1952 it made it into the first edition of the Diagnostic and Statistical Manual of Mental Disorders (now known as DSM-I) (American Psychiatric Association, 1952). Simple schizophrenia is described in the current ICD-10 in the following terms:

\footnotetext{
'an insidious but progressive development of oddities of conduct, inability to meet the demands of society, and decline in total performance. Delusions and hallucinations are not evident ... "negative" features of residual schizophrenia ... develop without being preceded by any overt psychotic symptoms. . . . the individual may [appear] self-absorbed, idle, and aimless' (World Health Organization, 1992: p. 95).
}

Although accepted in internationally agreed diagnostic systems, the diagnosis remained controversial. Reviewing the description in seven textbooks published between 1919 and 1978, Black \& Boffeli (1989) pointed out that a lack of drive and a deteriorating course are the only consistent diagnostic features. In particular there is a lack of agreement on the presence or absence of thought disorder as a diagnostic feature.

In an influential paper Stone et al (1968) trenchantly criticised the whole concept of simple schizophrenia on a number of grounds. First, in almost 40 years only one significant clinical paper, that by Kant in 1948, had been published on simple schizophrenia; second, the diagnosis (based on state hospital figures) was rarely used in practice; third, the diagnostic criteria were vague; and fourth, there was lack of agreement on the presence or absence of thought disorder. A decade later, the International Pilot Study of Schizophrenia (World Health Organization, 1979) failed to support the diagnostic utility of any of the sub-syndromes of schizophrenia, including the simple schizophrenia subtype.

Thus, the concept of simple schizophrenia disappeared in the third revision of the DSM and, although it remains within the ICD, ICD-10 advises that it be used with caution as a diagnosis of uncertain nosological validity.

Black \& Boffeli revisited the controversy in 1989 (Black \& Boffeli, 1989). They acknowledged the problems of heterogeneity but argued that the concept remained of heuristic value and was not covered by any of the existing categories in DSMIII. In a further paper (Black \& Boffeli, 1990) they reviewed the notes of 52 people diagnosed with simple schizophrenia between 1939 and 1964, reassigning diagnoses where they felt it necessary. 
Applying DSM-III criteria they found that these individuals were largely young, unemployed and living with their parents, but there was a great deal of heterogeneity with respect to diagnosis. The most common reassigned diagnosis was that of affective disorders. This was in obvious contrast to much of the literature, which emphasised the links between simple schizophrenia and other schizophrenic syndromes. They concluded, however, that the exclusion of simple schizophrenia from DSM-III had led to a diagnostic gap, with individuals who experienced loss of motivation and a progressively deteriorating course not falling within the boundaries of the diagnosis of a personality disorder but excluded from the diagnosis of schizophrenia. Arising from this they proposed an operational definition of simple schizophrenia, which they renamed 'simple deteriorative state'.

The DSM-IV (American Psychiatric Association, 1994) includes simple schizophrenia again as 'criteria set for further study' and names it simple deteriorative disorder, in line with the suggestion of Black \& Boffeli (1989). However, the criteria in DSM-IV differ from those proposed by Black \& Boffeli in at least one important particular. Although Black \& Boffeli emphasised the importance of functional decline, they suggested the inclusion of criteria relating to vague speech, odd beliefs and peculiar behaviours. The DSM does not include disorganised speech and behaviour.

\section{Studies of simple schizophrenia}

Perhaps understandably in view of its uncertain diagnostic validity, relatively few studies have focused on people with this diagnosis. Dworkin (1992) compared individuals fulfilling Bleuler's criteria for simple schizophrenia with individuals with typical schizophrenia. He re-examined the case histories of 151 monozygotic twin pairs with respect to negative symptoms, premorbid social adjustment and age at onset. He found that 220 individuals had a diagnosis of schizophrenia, 18 of whom met criteria for simple schizophrenia. Of these, 14 were co-twins. Dworkin concluded that individuals in the sample without hallucinations or delusions and those with typical schizophrenia were more similar than different. The main difference he did find was that fewer cases meeting the criteria for simple schizophrenia recorded a particular age at onset. This is consistent with the insidious onset described by Bleuler. Although not specifically commented on by Dworkin, it is interesting that such a high proportion of those with simple schizophrenia were co-twins, as this suggests that individuals with this disorder are less likely to be primarily identified as having schizophrenia and that it may represent a phenotype of schizophrenia in which the genetic contribution is particularly marked.

The importance of the genetic contribution to the simple schizophrenia subtype was further explored in two papers from the Roscommon Family Study. The first (Kendler et al, 1994) suggested that simple schizophrenia is relatively rare (they estimated a population prevalence of 5.3 per 10000$)$, debilitating and similar to typical schizophrenia in terms of presentation and course, the major difference being the presence or absence of psychotic symptoms. There was a higher than expected incidence of schizophrenia in relatives. In the second study, Kirkpatrick et al (2000) found the rate of schizophrenia in the families of individuals with a deficit syndrome (simple schizophrenia) to be similar to that for relatives of those with non-deficit-type schizophrenia, again suggesting a continuum between simple schizophrenia and the typical schizophrenia subtypes.

The criteria proposed by Black \& Boffeli (1989) were applied by Serra-Mestres et al (2000) to identify a sample of nine patients with simple schizophrenia. Psychometry and functional neuroimaging of this group revealed deficits consistent with the pattern seen in typical schizophrenia, including general intellectual impairment with deficits in memory and executive functioning. Most computed tomography scans were normal but a high rate of abnormality was found on single photon emission tomography.

Galderisi et al (1999) compared neuromorphological abnormalities revealed on MRI scans in individuals with simple schizophrenia, typical schizophrenia and controls. They concluded that both simple schizophrenia and typical schizophrenia show significantly more abnormalities than the control condition, specifically greater brain development abnormalities and greater ventricular and subarachnoid space volumes.

There has been very little discussion in the literature regarding treatment of simple schizophrenia. In our experience, Mr B responded moderately well to treatment with risperidone, showing both objective and subjective improvements. This is echoed by a handful of case reports recording successful use of atypical antipsychotics in treating this patient group (Hirose, 2000; Rollnik et al, 2001; Sekine et al, 2001).

\section{A dimensional approach}

We have illustrated the diagnostic complexities of simple schizophrenia with the case of a young man with normal premorbid development who has developed a deteriorating disorder in the absence of positive symptoms of schizophrenia. Within current classification systems, simple schizophrenia would 
seem to be the most apposite diagnosis. The problems and controversies associated with this diagnosis, however, remain. It has been argued that newer nosological systems for schizophrenia should be on a dimensional rather than categorical basis.

Liddle (1987), for example, proposed the three dimensions of psychomotor poverty, disorganisation and reality distortion as a more useful sub-syndrome division of chronic schizophrenia than the traditional positive-negative dichotomy. This proposal grew out of factor analysis of symptoms in patients with chronic illness but has proved influential and has been supported by neuroimaging findings (Liddle, 1996). Simple schizophrenia would be characterised as a relatively pure form of the psychomotor poverty syndrome because of the importance attached to negative symptoms.

However, we have described how successive classification systems have been unclear about the diagnostic importance of thought disorder. This may in part reflect the difficulty sometimes of characterising or quantifying disorganisation of thoughts using the language of descriptive psychopathology. The problems revealed by the more function-based assessments of neuropsychology and the assessment of living skills are more striking and persuasive. We would argue that Liddle's dimensional characterisation of schizophrenia would contribute towards preventing patients such as Mr B falling between diagnostic stools, representing as he does a relatively pure form not of psychomotor poverty but of disorganisation.

\section{Conclusions}

There exists a group of patients who present to psychiatrists with a pattern of insidious cognitive decline in the absence of florid positive symptoms of schizophrenia. Cognitive symptoms, including disorders of thought, may be overlooked owing to overreliance on the symptoms and signs of descriptive psychopathology. Functional assessments using psychological batteries and occupational therapy assessments may be more revealing. The dimensional classification of schizophrenia may help us identify and diagnose this group of patients by highlighting domains of social decline and cognitive disorganisation. Their illness is distinct from developmental disorders such as Asperger syndrome and attention-deficit hyperactivity disorder. As early treatment may be important, psychiatrists should be prepared to consider the diagnosis of simple schizophrenia, and psychiatric nosologies must allow for a category of relatively pure disorganisation, either by including disorganisation in the criteria for simple schizophrenia or by adopting a more dimensional approach to classification.

\section{References}

American Psychiatric Association (1952) Diagnostic and Statistical Manual of Mental Disorders. Washington, DC: APA.

American Psychiatric Association (1994) Diagnostic and Statistical Manual of Mental Disorders (4th edn) (DSM-IV). Washington, DC: APA.

Black, D. W. \& Boffeli, T. J. (1989) Simple schizophrenia: past, present and future. American Journal of Psychiatry, 146, 1267-1273.

Black, D. W. \& Boffeli, T. J. (1990) Simple schizophrenia: revisited. Comprehensive Psychiatry, 31, 344-349.

Burgess, P. W. \& Shallice, T. (1997) The Hayling and Brixton Tests. Bury St Edmunds: Thames Valley Test Company.

Coughlan, A. \& Hollows, S. (1995) Adult Memory and Information Processing Battery. Leeds: Psychology Department, University of Leeds.

Dworkin, R. H. (1992) Simple schizophrenia: an investigation of Bleuler's criteria based on the major twin studies of schizophrenia. Acta Psychiatrica Scandinavica, 85, 61-64.

Fisher, A. (2003) Assessment of Motor and Process Skills (5th edn). Fort Collins, CO: Three Star Press.

Galderisi, S., Bucci, P., Mucci, A., et al (1999) Simple schizophrenia: a controlled MRI and clinical/neuropsychological study. Psychiatric Research: Neuroimaging Section, 91, 175184.

Hirose, S. (2000) Effectiveness of risperidone in simple schizophrenia: single report. Journal of Clinical Psychiatry, 61, 300301.

Kant, O. (1948) Clinical investigation of simple schizophrenia. Psychiatric Quarterly, 22, 141-151.

Kendler, K. S., Mcguire, M., Gruenburg, A. M., et al (1994) An epidemiological, clinical, and family study of simple schizophrenia in county Roscommon, Ireland. American Journal of Psychiatry, 151, 27-34.

Kirkpatrick, B., Ross, D. E., Walsh, D., et al (2000) Family characteristics of deficit and nondeficit schizophrenia in the Roscommon family study. Schizophrenia Research, 45, 57-64.

Kraepelin, E. (1919) (in translation) Dementia praecox and paraphrenia. In Textbook of Psychiatry (8th edn) (trans. R. M. Barclay). Edinburgh: ES Livingston.

Lezak, M. D. (1995) Neuropsychological Assessment (3rd edn). Oxford: Oxford University Press.

Liddle, P. F. (1987) The symptoms of chronic schizophrenia A re-examination of the positive-negative dichotomy. British Journal of Psychiatry, 151, 145-151.

Liddle, P. F. (1996) Functional imaging - schizophrenia. In Biological Psychiatry (ed. E. C. Johnstone). Oxford: Royal Society of Medicine Press.

Rollnik, J. D., Schneider, U. \& Emrich, H. M. (2001) Successful treatment of simple schizophrenia with atypical neuroleptics (clozapine, risperidol). Nervenarzt, 72, 380-383.

Sekine, Y., Takei, N., Iyo, M., et al (2001) Effective risperidone treatment for simple deteriorative disorder (simple schizophrenia): a case report. Schizophrenia Research, 50, 213-214.

Serra-Mestres, J., Gregory, C. A., Tandon, S., et al (2000) Simple schizophrenia revisited: a clinical neuropsychological, and neuroimaging analysis of nine cases. Schizophrenia Bulletin, 26, 479-493.

Stone, A. A., Hopkins, R., Mahnke, M. W., et al (1968) Simple schizophrenia-syndrome or shibboleth. American Journal of Psychiatry 125, 61-68.

Wechsler, D. (1997) Wechsler Adult Intelligence Scale (3rd UK edn). London: Psychological Corporation.

Wechsler, D. (2001) Wechsler Test of Adult Reading. London: Psychological Corporation

Wilson, B. A., Alderman, N., Burgess, P. W., et al (1996) Behavioural Assessment of the Dysexecutive Syndrome. Bury St Edmunds: Thames Valley Test Company.

World Health Organization (1948) The Sixth Revision of the International Classification of Diseases. Geneva: WHO.

World Health Organization (1979) An International Follow-Up Study of Schizophrenia. New York: John Wiley \& Sons.

World Health Organization (1992) The ICD-10 Classification of Mental and Behavioural Disorders: Clinical Descriptions and Diagnostic Guidelines. Geneva: WHO. 


\section{MCQs}

1 The diagnosis of simple schizophrenia can be made, according to ICD-10, if the following are present:

a persistent hallucinations, in any modality

b prominent psychomotor disturbances

c progressive decline in day-to-day functioning

d prominent affective changes

e illness duration of 6 months.

2 Simple schizophrenia was first described:

a in 1903

b by Kraepelin

c by Diem

$\mathrm{d}$ as a separate disease entity from dementia praecox

e as a subgroup of dementia praecox but lacking hallucinations and delusions.

3 The diagnosis of simple schizophrenia has been criticised on the grounds of:

a lack of use of the diagnosis in clinical practice

b heterogeneity of individuals diagnosed with simple schizophrenia

c symptoms that are more readily encompassed by classification within mood disorder

$\mathrm{d}$ disagreement whether or not thought disorder is consistent with the diagnosis

e not having a pathophysiological basis.

4 Black \& Boffeli criteria for simple deteriorative disorder:

a are adopted by DSM-IV as the basis of simple deteriorative disorder

b were formulated as an alternative to simple schizophrenia

c highlight functional decline

d exclude vague speech, odd beliefs and bizarre behaviours

e include thought disorder.

5 A dimensional approach to schizophrenia:

a was proposed by Liddle

$\mathrm{b}$ is included in the standard ICD-10 classification

c includes dimensions of psychomotor poverty, disorganisation and reality distortion

d would describe the clinical syndrome of simple schizophrenia as reality distortion

e would do away with the need for phenomenology.

\section{NEW OUT FROM GASKELL}

\section{So Young, So Sad, So Listen (second edition)}

\section{By Philip Graham and Carol Hughes}

Has your daughter lost her sparkle? Has everyday life become a trial for your son? As a teacher, do you think that difficult, aggressive 11-year-old boy has other problems and wonder why that 14-year-old girl is so withdrawn? The problem could be childhood depression, a common but often treatable condition.

Illustrated by cartoons, this book is intended to help parents and teachers of depressed children and teenagers. Young people themselves will also find it useful. As should school nurses, social workers, health visitors, family doctors and anyone who works with children. This second edition contains a foreword by multi-award-winning author Philip Pullman.

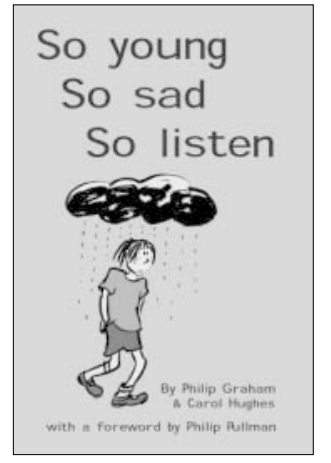

The aim is to help those involved to recognise the signs of depression in children and to understand possible causes. The authors give practical advice and information about the support and help you can give. Above all, they show that you can help and that, given the chance, that spark may well return!

\section{Aug 2005, Paperback, 64 pages, Price $£ 7.50$ ISBN 1904671233}

\section{MCQ answers}

$\begin{array}{llllll}\text { 1 } & & \text { 2 } & 3 & 4 & 5 \\ \text { a F } & \text { a T } & \text { a T } & \text { a F } & \text { a T } \\ \text { b F } & \text { b F } & \text { b T } & \text { b T } & \text { b F } \\ \text { c T } & \text { c T } & \text { c F } & \text { c T } & \text { c T } \\ \text { d F } & \text { d F } & \text { d T } & \text { d F } & \text { d F } \\ \text { e F } & \text { e T } & \text { e F } & \text { e T } & \text { e F }\end{array}$

AVAILABLE FROM:

Book Sales, Royal College of

Psychiatrists, 17 Belgrave Square, London SW1X 8PG, UK.

Tel: +44 (0)20 72352351 ext 146. Fax: +44 (0)20 72451231 .

Website: www.gaskell-books.com 